\title{
ENLACE: ¿Una alternativa real para la evaluación de comprensión lectora?
}

\author{
(ENLACE: A Real Alternative for the Evaluation of \\ Reading Comprehension?)
}

\begin{abstract}
LAUra Aurora Hernández RAMÍREz ${ }^{\mathbb{D}}$, Universidad de Tlaxcala, Tlaxcala, México ADRIANA Rosales Rodríguez, Universidad autónoma de Tlaxcala, Tlaxcala, México Claudia Galicia Solís, Universidad Tecnológica de Tlaxcala, Tlaxcala, México
\end{abstract}

\author{
Volumen 1, Número 1 \\ Edición especial. Febrero de 2016
}

$$
\text { p. } 26-47
$$

Este número se publicó el 5 de febrero de 2016

Artículo recibido: 19 de enero de 2015

Artículo aprobado: 10 de noviembre de 2015

ISSN: 2448-5942, doi: https://doi.org/10.36799/el.v1i1.11

\section{Citar este artículo como:}

Hernández Ramírez, L. A., Rosales Rodríguez, A., y Galicia Solis, C. (2016). ENLACE: ¿Una alternativa real para la evaluación de comprensión lectora? Estudios גambda. Teoría y práctica de la didáctica en lengua y literatura, 1(1), 26-47. https://doi.org/10.36799/el.v1i1.11

Derechos de autor: El autor o autores conservan en todo momento sus derechos morales y patrimoniales sobre la obra; la obra no se puede alterar, transformar o ampliar; siempre debe reconocerse la autoría del documento

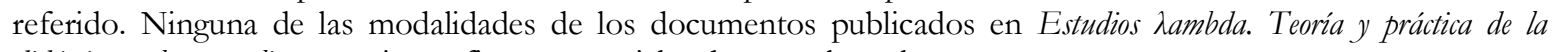
didáctica en lengua y literatura tienen fines comerciales de naturaleza alguna.

Los contenidos de este artículo están bajo la licencia de Creative Commons Atribución No Comercial- Sin Derivadas 4.0 Internacional @) 


\title{
ENLACE: ¿Una alternativa real para la evaluación de comprensión lectora?
}

\author{
(ENLACE: A Real Alternative for the Evaluation of Reading Comprehension?)
}

\author{
LAURA AURORA HERNÁNDEZ RAMÍREZ ${ }^{1}$ \\ ADRIANA ROSALES RODRÍGUEZ ${ }^{2}$ \\ Claudia Galicia Solís ${ }^{3}$
}

\begin{abstract}
RESUMEN
Hasta hace un par de años, la prueba ENLACE se había posicionado en el sistema educativo mexicano como el medio oficial y punto de referencia para la evaluación de competencias en comprensión lectora y matemática, así como de los conocimientos relacionados con otros campos disciplinares (historia, ciencias, etc.). A principios de 2014, la perspectiva cambió al "posponer" la prueba, argumentando múltiples errores e inadecuaciones en su aplicación, así como de presuntos actos de corrupción relacionados con el manejo y difusión de sus resultados (Backhoff Escudero, 2014). No obstante, esto se sigue asumiendo que tener un bajo puntaje en ENLACE significa necesariamente "no ser un buen lector". En este artículo ponemos en tela de juicio esta creencia, pues desde las teorías discursivas fundadas en la literacidad, la comprensión académica de los textos es un proceso dinámico de naturaleza socio-semiótica, cuya totalidad y complejidad no puede ser "medida" por ejercicios evaluativos de naturaleza cuantitativa como lo es Enlace. Para evidenciar con mayor claridad el alcance de la prueba, analizamos el contenido de las preguntas y del modelo de lectura que en ellas subyace; para ello, se les aplicó a cuatro grupos de universitarios una batería de comprensión lectura correspondiente al examen de educación media superior del 2010 para probar su idoneidad en la determinación de la aptitud y nivel de comprensión de quien realiza la prueba. Fue posible constatar que algunos de los errores en que pueden incurrir los examinados no dependen de su habilidad lectora, sino del diseño mismo de la prueba, de las condiciones de aplicación, a la falta de claridad en la pregunta y/o en las respuestas que se ofrecen a los lectores, todo esto motivado por un concepto muy limitado de lo que abarca el fenómeno de la comprensión discursiva.
\end{abstract}

PALABRAS CLAVE: Prueba Enlace, comprensión lectora, exámenes estandarizados, enfoque sociocognitivo y discursivo de la lectura.

\begin{abstract}
Two years ago ENLACE test was stablished as the official instrument and point of reference for the evaluation of competences in Reading comprehension and Mathematics, and also in the knowledge related to other disciplines (history, sciences, etc.) At the beginning of 2014, the perspective changed when "the test was put off due to so many errors and inadequacies in its application, and also due to assumed corruption acts related to handling and publishing its results (Bakhoff Escudero, 2014). This is still assumed: having a low percentage in ENLACE means necessarily "being not a good reader." In the present article that belief is questioned, so since discursive theories based on literacy, academic comprehension of texts is a dynamic process of socio-semiotics quality whose academic totality and complexity should not be "measured" by means of evaluation exercises, quantitative in nature, as happens with ENLACE test. In order to provide more clarity in the reach of the test, we analyzed the underlying contents of questions and reading models used. With this objective in mind, four groups of university students were asked to answer a set of reading comprehension equivalent to the test of preparatory school in 2010, in order to prove its suitability in determining aptitude and level of comprehension of the person who does the test. We were able to confirm that some errors on which students may incur, depend not on their reading ability but on several other factors: design of the test itself, conditions of application, vagueness of questions and/or model answers provided to readers. All this is motivated by a very limited concept of what is covered by the phenomenon of discursive comprehension.
\end{abstract}

KEYWORDS: Enlace test, reading comprehension, standardized exams, sociocognitive and discursive reading aproach.

${ }^{1}$ Mtra. en Ciencias del Lenguaje docente de la Universidad de Tlaxcala, laurora711@gmail.com

2 Maestrante en Lenguas Modernas y Estudios del Discurso de la Universidad Autónoma de Tlaxcala, jefa r r@hotmail.com

${ }^{3}$ Maestrante en Lenguas Modernas y Estudios del Discurso de la Universidad Tecnológica de Tlaxcala claudia216 50@hotmail.com

Artículo recibido: 19 de enero de 2015

Artículo enviado a corrección: 24 de septiembre de 2015

Aprobado: 10 de noviembre de 2015

Hernándeæ, Rosales y Galicia doi: https://doi.org/10.36799/el.v1i1.11 Volumen 1, Número 1, Año 2016, ISSN: 2448-5942 
"Les decimos a los niños que leer es divertido, que es entretenido, que es hermoso, pero no les permitimos disfrutar de la lectura, sino que empañamos ésta con interrogatorios impertinentes y con exámenes y reportes de lectura que nada prueban pero que sí consiguen hacerlos sentir incapaces de comprender e ineptos para expresarse"

Juan Domingo Argüelles. (2011). Estás leyendo... ¿y no lees? Un libro contra la obligación de leer, p. 52.

INTRODUCCIÓN. Antecedentes, objetivos y planteamiento del problema

Es a principios de este siglo que la evaluación masiva de las competencias discursivas de los estudiantes de los diversos niveles de educativos en México, se convirtió en un tema de importancia social y política. Desde entonces, los resultados del examen estandarizado PISA (Programme for International Student Assesment) en habilidades verbales y matemáticas de aplicación internacional, auspiciado por la OCDE, se ha convertido en una referencia obligada cuando se habla de evaluación de comprensión lectora. Según Vidal (2009), este ejercicio evaluativo, representó la primera medición confiable de nuestro sistema educativo y fue la motivación para que en nuestro sistema educativo se instauraran proyectos de esta naturaleza que diagnosticaran diferentes aspectos y niveles relacionados con los logros educativos a nivel colectivo o individual.

La prueba ENLACE (Evaluación Nacional del Logro Académico en Centros Escolares) nació en 2006 como uno de estos proyectos cuyo objetivo era diagnosticar en la Educación Básica y en la Educación Media Superior (EMS, en adelante), la capacidad de los estudiantes para aplicar conocimientos y habilidades básicas (comprensión lectora y matemática) a situaciones reales para hacer un uso adecuado de la lengua (SEP 2013), y el rendimiento básico en una tercera asignatura rotativa. Tras ocho años de aplicación, en febrero de 2014, las autoridades educativas declararon la suspensión de la prueba argumentando la manipulación indebida y perversa que de los resultados se hicieron en las últimas aplicaciones (Vaca y Montiel Guerra 2008), así como al manejo político al que se sometieron los resultados de estas evaluaciones, 
factores que propiciaron que dejaran de ser confiables, y por lo que "los supuestos avances son más una ficción que una realidad” (Beckoff Escudero 11).

La aplicación y utilidad de los exámenes estandarizados han sido puestos en discusión dentro de la investigación educativa en México y Latinoamérica, y el valor del Enlace como herramienta fidedigna de evaluación del proceso de comprensión lectora ha sido seria y reiteradamente cuestionado por especialistas (Sánchez Cerón 2013; Vaca y Montiel Guerra 2008). Incluso, se ha expuesto que la aplicación de estos exámenes difícilmente incrementará la calidad del sistema educativo (Díaz Barriga 2006).

Además, como sabemos, existen diversas posturas en el campo de la pedagogía, de la psicología y de la psicolingüística que proponen definir a la posibilidad de los lectores de relacionarse comprensiva y críticamente con un texto, entendido generalmente como comprensión lectora, por lo que los atributos que la caractericen como una habilidad, una competencia o una actividad socio-semiótica (Vaca y Montiel Guerra 2008; Parodi 2011), serán objeto de controversia, pero indudablemente la claridad con la que se le defina será un elemento clave para fundamentar cualquier actividad de evaluación, pues el concepto operativo con el que se prepare un proyecto de esta naturaleza, le dará la coherencia necesaria a la implementación del mismo. En este diseño tendrán que incluirse, por supuesto, el tipo preguntas que se harán, la naturaleza de las respuestas idóneas que se esperan y la definición de los alcances de la evaluación resultante.

Por ello, nuestro interés ha sido integrarnos a esta discusión desde la perspectiva de la enseñanza de la lengua materna, basadas en metodologías en las que el género discursivo (Hernández Ramírez 2010a y b) se constituye en el concepto principal de organización del ciclo pedagógico. Asimismo, estamos convencidas de la necesidad de articular visiones interdisciplinarias que concreten nuevos proyectos de evaluación, estandarizados o no, cuyo interés sea guiar con mayor sistematicidad el desarrollo de las políticas y acciones educativas dentro y fuera del aula en cuanto a enseñanza del lenguaje se refiere. Así pues, argumentamos que hacer un diagnóstico del nivel de comprensión lectora de la población escolar en los diferentes niveles educativos en México implica mucho más que responder un cuestionario elaborado a partir de un concepto de naturaleza cognitiva aislable, como el que parece predominar en el diseño de las pruebas estandarizadas actualmente vigentes, incluyendo la de 
PISA. En la postura que representamos, en cambio, la comprensión se entiende como un ejercicio semiótico de intrínseca naturaleza social, cognitiva y lingüística (Parodi 2011), en donde para evaluar la idoneidad del proceso es fundamental haber definido claramente el objetivo, el contexto disciplinar o cotidiano, las características específicas del lector con el fin de delinear con mayor sistematicidad los parámetros o categorías que podrían aproximarse a definir y “dimensionar" la comprensión en una situación específica.

De esta manera, señalaremos algunas observaciones sobre el diseño de los reactivos sobre el texto expositivo en la prueba de comprensión lectora involucrada en una versión de práctica del año 2010 de la prueba Enlace para EMS en ese año. Estas reflexiones se originaron en un curso del posgrado de Lenguas Modernas y Estudios del discurso de la Facultad de Filosofía y Letras de nuestra universidad al abordar el estado del arte en cuanto al tema de evaluación de las habilidades y competencias relacionadas con el lenguaje.

\section{METODOLOGÍA DE TRABAJO}

Para aproximarnos al problema señalado, aplicamos a un grupo de posgrado (estudiantes y docente) en la especialidad de análisis del discurso -cuyos miembros ya han demostrado capacidad de análisis e interpretación de textos de especialidad- el cuestionario del texto Ciudad entre nubes, como ejemplo de un texto expositivo, disponible como un ejercicio de preparación en el portal destinado a la prueba (http://www.enlace.sep.gob.mx/). En una primera fase, hicimos un análisis sobre las dificultades al ser contestada la prueba por medio de un cuestionario aplicado a los participantes. Para reforzar estas primeras apreciaciones, se realizó el mismo ejercicio en otros tres grupos de estudiantes universitarios en licenciatura: dos grupos de enseñanza de lenguas de nuestra universidad y a un grupo de estudiantes de una universidad tecnológica del Estado de Tlaxcala. En esta fase, se tomó como punto de referencia para la reflexión el conjunto de preguntas en donde el porcentaje de error fue mayor y con base en esta evidencia expusimos una serie de hipótesis sobre los procesos discursivos que podrían motivar estas respuestas “erróneas”, según la concepción tradicional, pero que, en algunos casos puede evidenciar, desde nuestra perspectiva, las limitaciones del modelo bajo el cual se entiende la interpretación y la reflexión en estas pruebas estandarizadas.

Hernández, Rosales y Galicia doi: https://doi.org/10.36799/el.v1i1.11 Volumen 1, Número 1, Año 2016, ISSN: 2448-5942 
Así pues, revisamos en la bibliografía oficial disponible (Ceneval 2011 y 2012; SEP 2013) el concepto de comprensión lectora con el cual los especialistas elaboraron los ejercicios y reactivos que medían el grado de habilidad de los que contestaron el examen y lo confrontamos con un concepto sociosemiótico y basado en la literacidad (Parodi 2011) que no es ajeno, según los manuales correspondientes, a los lineamientos que se siguen en los actuales programas de estudio de educación básica y media superior.

De esta forma, en la discusión que presentamos a continuación destacamos tres aspectos relacionados que evidencian una interpretación reduccionista de la comprensión, pues una respuesta incorrecta en este tipo de exámenes no necesariamente significa no saber leer ni comprender, a saber: a) la concepción del lenguaje y la habilidad lectora, b) los niveles y características en los que se organiza la actividad de comprensión de estas pruebas, y c) la problemática surgida a partir de una clasificación textualista de los textos escritos que es la que predomina en los manuales técnicos mencionados.

En la siguiente sección, tomando como base las respuestas con mayor porcentaje de error, describimos e interpretamos las dificultades que los lectores pueden experimentar al resolver las preguntas señaladas desde una visión discursiva basada en la literacidad del proceso de comprensión. Estas explicaciones nos dan un indicio de que los errores pueden deberse alternativamente a una inadecuación del propio diseño del examen, de las características propias de la actividad lectora, etc., como lo mostraremos más adelante.

Como colofón del trabajo, plantearemos algunas conclusiones que pueden servir como hipótesis de trabajos posteriores y algunas recomendaciones que pudieran ser útiles a quienes se interesen en construir ejercicios evaluativos que den mayor certidumbre sobre la actividad de la comprensión de lectura académica.

\section{CONCEPCIÓN Y FUNCIÓN DEL LENGUAJE EN LA COMPRENSIÓN DISCURSIVA}

En el manual técnico de la prueba que corresponde al texto Ciudad entre nubes (CENEVAL 29), se define a "la Habilidad Lectora como la capacidad de un individuo para comprender, utilizar, analizar textos escritos, con el fin de alcanzar sus propias metas, desarrollar el conocimiento y

Hernández, Rosales y Galicia doi: https://doi.org/10.36799/el.v111.11 Volumen 1, Número 1, Año 2016, ISSN: 2448-5942 
el potencial personal, y participar en la sociedad”. En la versión 2014, cuando el currículo se encuentra dentro de un enfoque por competencias, la definición anterior se repite, aunque el término habilidad se sustituyó por comprensión lectora como la capacidad para utilizar textos y ampliar la competencia comunicativa:

...mediante la identificación de su estructura, sus funciones y sus elementos (de los textos) y para desarrollar una competencia comunicativa para construir nuevos conocimientos que le permitan intervenir activamente en la sociedad (Ceneval 29).

Estas definiciones muestran, como lo ha discutido Vaca (2008), una traducción desafortunada y basada en concepciones ya superadas por la psicología de las definiciones proveídas por la OCDE. El autor mencionado insiste en que el tipo de definición de la lectura como una habilidad o capacidad centrada en el individuo es coherente con una visión que supone es posible aislar, y por lo tanto medir, las funciones integrales del pensamiento (Vaca y Montiel Guerra 2008).

Leer se considera un proceso porque implica la coordinación de diferentes habilidades y conocimientos $[\ldots]$ antes, durante y después del momento de leer. Es muy importante que el lector vaya construyendo una comprensión de la lengua que integre su aspecto morfológico y funcional, lo primero se refiere a las consideraciones formales del lenguaje que, como sistema debe respetar signos, reglas y convenciones para ser entendido (Ceneval 29).

Asimismo, podemos notar en esta definición que se está considerando que la comprensión se está circunscribiendo al momento de la lectura; además, proyecta al lenguaje como un sistema organizado como una dicotomía entre un sistema formal normativo, cuyas convenciones se deben respetar, sin aclarar a qué tipo de reglas se refiere, y un aspecto funcional en el que no se define su relación con el proceso comprensivo, como si esta fuera una relación lineal no conflictiva. Parece, entonces, que aún se está conceptualizando al lenguaje básicamente como un sistema normativo y a la comprensión como un proceso independiente de una estructura social y de procesos semióticos que incluyen los cognitivos, pero también los ideológicos, componentes que están más allá del momento en que el lector realiza la lectura.

Hernándeそ, Rosales y Galicia doi: https://doi.org/10.36799/el.v1i1.11 Volumen 1, Número 1, Año 2016, ISSN: 2448-5942 
Así pues, desde una perspectiva discursiva el hablar de comprensión lectora como un concepto único y homogéneo en el que se concreten todas las maneras que reviste la comprensión humana a través del lenguaje tiene que ser superado. Los estudios de análisis del discurso y la literacidad de los últimos veinte años dentro de la lingüística ha hecho posible definir a la lectura como un proceso de naturaleza social, cognitivo, semántico, dinámico y complejo, lo cual tendría que plantear que la comprensión también implica un saber comprender y, por lo tanto, un aprendizaje de la comprensión, así como una diversidad de tipos de "comprensión", a los que los hablantes tienen que incorporarse, pues se comprenden muchos tipos de información o procesos en situaciones diversas y a través de diversos géneros o formatos. Por lo tanto, podemos afirmar la naturaleza diversa y dinámica de la comprensión, es decir, la comprensión nunca es abstracta, sino siempre está situada y retomamos a Parodi:

De lo dicho hasta aquí se desprenden la existencia de varios factores que influyen en el proceso de la lectura [...] la naturaleza del signo, tipo de significación (relación natural o arbitraria entre ambas caras). Otros factores, en cambio, dependen del sujeto lector, sus estrategias de lectura (generalmente asociadas a la edad del sujeto lector), su conocimiento previo y el contexto cultural [...] otro factor relacionado con el sujeto y que resulta fundamental para determinar las características del proceso lector. Nos referimos al objetivo o propósito con el que el sujeto emprende la lectura de un texto [Las cursivas son nuestras] (55).

De los diversos objetivos que el autor y la bibliografía especializada señalan como objetivos lectores (leer para informarse, leer para entretenerse, para pasar el tiempo, leer para aprender, etc.) cabría preguntar qué propósito se plantea en las lecturas de Enlace, pues este parámetro metodológico no se establece con claridad en la prueba, pues se insiste en que la lectura es una herramienta útil, tanto en la vida social como base del aprendizaje, pero asumir cualquiera de estas opciones definirá un proceso diferente de comprensión. Es posible asumir que en los ejercicios para la EMS, la pretensión es que el estudiante se ubique en una lectura de aprendizaje, es decir, en una lectura y comprensión académicas, lo que restringe y especifica las mejores condiciones para realizar la prueba. Esta precisión nos permite plantear algunas consideraciones relacionadas con el tema. 
Por ejemplo, las condiciones idóneas para realizar una lectura de este tipo no son aquellas en las que se aplica la prueba. En primer lugar, el tiempo que fue necesario por parte de lectores expertos en lectura académica (docente y estudiantes de posgrado) para resolver el examen aplicando las estrategias necesarias para encontrar la respuesta correcta esperada, fue al menos de 2 horas, considerando que el texto debe leerse varias veces y que el orden en que se encontraban las respuestas a las preguntas formuladas en el ejercicio mencionado no estaban en orden secuencial; es decir, había que localizar y recuperar la información necesaria para la construcción de la inferencia en diversos lugares del texto sin un orden secuencial, por lo que localizar la sección en la que había que trabajar no siempre fue rápida ni inmediatamente relacionada con la respuesta anterior. De esta forma, es posible suponer que quien resuelve el examen sabe del tiempo limitado (45 minutos, según el manual) disponible para contestar los 17 reactivos de cada sección y que, por día realizará cuatro sesiones (dos de matemáticas y dos de lectura) por lo que necesita responder sin dilaciones, condición que, paradójicamente, plantea el disponer de poco tiempo para la reflexión. Este tipo de condiciones, además, crean la imagen falsa de que la lectura analítica, como lo es la académica, es un proceso expedito, memorístico, rutinario; idea, como sabemos, está muy alejada de las prácticas académicas auténticas.

Otro aspecto mencionado por el manual técnico es que “...la lectura [...] involucra distintas habilidades que se adquieren en los primeros años de escuela y se fortalecen en la vida cotidiana" (Ceneval 28). En este juicio, encontramos dos inconsistencias relacionadas con esta concepción homogeneizadora de la comprensión: que estas habilidades se adquieren en un solo periodo crítico de la vida del hablante y, en segundo lugar, de que la vida cotidiana reforzará un proceso, el académico, que no se vive en esta fase de la vida del individuo. La lectura académica tiene su ámbito natural en la vida académica escolar; además, se enseña y se fortalece no solo en el inicio de la vida escolar sino debe renovarse a lo largo de las diversas etapas del desarrollo académico. Podemos establecer que el niño, el adolescente o el adulto tendrán que ir atravesando por diversos estadios de comprensión discursiva a medida que confrontan el reto de involucrarse y participar activamente en diversas comunidades de práctica (Wenger 2001). Digamos que con la potencialidad comprensiva se nace, pero las comprensiones concretas se construyen básicamente a través de un aprendizaje constructivo 
social. Como prueba de esto se encuentran las importantes propuestas teórico-metodológicas en alfabetización académica (Carlino 2013), que se han desarrollado desde inicios del presente siglo, proyectos en donde se ha insistido en la necesidad de implementar programas de intervención específicos, en el caso de la educación superior, por ejemplo, para preparar a los estudiantes ante las nuevas y diferentes maneras de significar en este nivel educativo (Castro Azuara, et al. 2013). Asimismo, Parodi (37) señala: “...el desarrollo de la capacidad de trascender la realidad que rodea al sujeto, aunque es innata, evoluciona dependiendo del aprendizaje".

De estos puntos podemos subrayar dos aspectos de un proceso comprensivo que es necesario recordar cuando se trata de proponer actividades de evaluación: su naturaleza sociocognitiva discursiva y, por lo tanto, su carácter situado y dinámico y, que la evolución hacia estadios de comprensión académica depende de acciones pedagógicas concretas, sistemáticas, secuenciales y transdisciplinares, cuya planeación global no recae únicamente en los docentes. Por ello, asumimos que si en las evaluaciones los estudiantes no poseen o no demuestran los niveles deseables la razón es porque no se les ha enseñado a comprender académicamente. Estamos, entonces, ante un problema que no debe señalar como principal responsable al estudiante, ni siquiera al maestro cuyo poder de decisión curricular en la educación obligatoria es limitado, sino a un sistema que no ha incorporado una metodología sistemática ni clara sobre la literacidad académica en sus diferentes niveles educativos. Por ejemplo, en el tiempo de un currículo basado en competencias, señalar al conteo de palabras por minuto o al número de libros leídos por estudiante como una estrategia válida para desarrollar lectores críticos, no puede ser menos que contradictoria y, por ello, equivocada y reduccionista (Merino 2013).

\section{NIVELES DE COMPRENSIÓN: LA MEDIDA DE LA REFLEXIÓN Y LA INTERPRETACIÓN}

En Enlace, al igual que en la prueba PISA, se definen tres niveles de complejidad en los procesos cognitivos implicados en la comprensión lectora, a saber: la extracción de datos, la interpretación, relacionadas con procesos inferenciales de diversos niveles, y en el nivel de

Hernández, Rosales y Galicia doi: https://doi.org/10.36799/el.v1i1.11 Volumen 1, Número 1, Año 2016, ISSN: 2448-5942 
mayor complejidad, a la reflexión que debe realizar el lector, ya sea acerca de la forma del texto o del contenido, todos medidos con una escala que los clasifica en niveles de insuficiente, básico, bueno o excelente.

El primer nivel que se evalúa, el de la extracción de información, es el que, según nuestro ejercicio, representa en estas pruebas el mejor evaluado. La mayoría de los estudiantes suele localizar la información sin mayores problemas, por lo que estas pruebas parecen ser útiles para este tipo de comprensiones muy concretas relacionadas con estrategias memorísticas. Sin embargo, cuando se trata del nivel inferencial y reflexivo las dificultades se incrementan, pues en estos niveles es necesario desarrollar una serie de estrategias metacognitivas relacionadas con los conocimientos previos sobre el tema, el contexto social y/o sobre el papel de los textos en los procesos de interacción, estrategias que presumiblemente no ha desarrollado. El estudiante no ha sido inducido a concebirse como un interlocutor activo de los textos, sino como un receptor pasivo de la información y, debido a la mayoría de las prácticas reduccionistas de copiado, de dictado a las que se ha enfrentado continuamente, entiende que su tarea se circunscribe a vaciar esta información con fines de obtención de calificaciones, pero sin ninguna utilidad real en el medio social.

Asimismo, estos niveles inferenciales y de reflexión en su mayoría responden, como se enfatizó en el apartado anterior, a procesos que no son de naturaleza innata y, aún menos, suponen una relación transparente e inmediata con la discursividad. Es decir, entender ideas y poder expresarlas a través del lenguaje de manera oral o por escrito, no es un proceso expedito ni solipsista. El conseguir ser crítico y aprender por medio de la lectura requiere integrar en un proceso una serie de operaciones y estrategias de diversa naturaleza (lingüística, textual, cognitiva, social, ideológica, semiótica). Parodi (2011), por ejemplo, resalta dos dimensiones de lectura (la literal y la relacional, en donde se vincula lo explícito con lo no explícito), dos niveles de información textual (superficial e implícita y profunda), inferencias de tipo textual, tales como las relaciones cohesivas hasta relaciones lógicas entre los diversos aspectos del texto, y dos tipos de aprendizajes a partir de la lectura (la memorización y la construcción relacional, la reconstrucción de una representación de un universo). Además, el autor (Ibid) explica cómo en esta dimensión la interpretación se construye a través de la integración de una red de 
intersubjetividades que consiste en establecer un circuito de al menos cinco fases de puesta en común con otros lectores para poder construir un conocimiento profundo del texto.

A diferencia de este proceso multidimensional y dinámico, Enlace enfrenta a los estudiantes a una suerte de ruleta en la que la interpretación y la reflexión implican una sola respuesta correcta, proveniente de una autoridad que se coloca como un modelo de interpretación y de reflexión unívoco, lo que contradice la naturaleza crítica de estos procesos, como lo ilustra el epígrafe de nuestro trabajo. Tal parece, por lo tanto, que el estudiante en esta prueba cumple un papel de consumidor de la información, no de un constructor de sentido que interactúa con el texto y con el contexto de la situación de lectura.

En resumen, creemos que el examen evidencia el tipo de operaciones cognitivas que tradicionalmente se han favorecido durante las prácticas de lectura dentro de las aulas: la localización de ítemes léxicos, la localización de información concreta en el subrayado, prácticas que se quedan en un concepto referencialista de la lectura, vista como una operación casi mecánica, no de construcción crítica de significado. Por todo esto, insistimos en que es necesario reforzar la relación de la lectura con la construcción de marcos de conocimientos útiles para el análisis del mundo social del estudiante, lo cual incluye, evidentemente, su vida académica.

\section{TIPOS TEXTUALES VERSUS GÉNEROS DISCURSIVOS}

Enlace establece una clasificación de los textos en cuatro tipos que suponen una clasificación funcional. A continuación se parafrasean algunas de las características atribuidas a ellos (CENEVAL 29):

a) Apelativo, texto en donde se trata de suscitar interés en el receptor o de convencerlo de actuar. Se relaciona con las acciones de seguir una instrucción, de convencer, y establece la definición en el manual que usa la forma imperativa o condicional del verbo. Da como ejemplo a la carta. 
b) Argumentativo, el texto que pretende demostrar un argumento o contrargumento e intenta convencer o hacer cambiar de idea. El artículo de opinión se señala como un ejemplo representativo.

c) Expositivo, representa la función referencial de los textos; es utilizado cuando se quiere expresar clara y objetivamente un mensaje. Está relacionado con las investigaciones científicas, por lo que el artículo de divulgación, acompañado de un glosario y una tabla se señala como el mejor ejemplo.

d) Narrativo. Cuento o microrrelato relacionado con la función poética, lo relaciona con la descripción subjetiva de la realidad por parte del escritor, en donde es importante lo que se dice y cómo se dice.

Así pues, estas definiciones están inspiradas en un esquema simplificado de la teoría estructuralista funcional de Roman Jakobson, que fue una aportación importante en los 60 del siglo pasado, pues fue de las primeras descripciones funcionales del rol del lenguaje en la comunicación. No obstante, debemos señalar que en la actualidad existen modelos que describen la dinámica de interacción lingüística con un mejor acercamiento de su dinámica social y cognitiva a partir de teorías de la enunciación y del análisis del discurso (Bolívar 2007; Martínez Solís 2002), en donde el lenguaje cumple tareas o prácticas sociales. En estos modelos enunciativos, en lugar de hacer uso de una clasificación textualista como la expuesta por las definiciones anteriores, se utiliza como concepto básico de clasificación de estas prácticas el concepto de género discursivo que ha sido discutido ampliamente y aceptado como una manera de organizar con mayor certidumbre los currículos escolares (Castro Azuara 2013; Hernández Ramírez 2010a y b) por mencionar algunas de las alternativas. El problema de las definiciones textualistas, sobre todo su versión escolar como las que se usan en los libros de texto y las citadas, es que se basan en el tipo de secuencia discursiva que caracteriza a un texto; por ejemplo, el texto expositivo pareciera que estaría únicamente conformado por secuencias descriptivo-explicativas, o el argumentativo sólo con secuencias argumentativas. Cuando se trata de textos sencillos o simples como los que regularmente son adecuados para niveles de educación básica, suele suceder así; sin embargo, cuando se trata de textos complejos, propios de la vida social o académica, como el texto Ciudad entre nubes, que se ubica dentro de los textos expositivos, contiene secuencias descriptivas y explicativas, pero también, diversas secuencias 
argumentativas; prueba de ello es que en una de las preguntas del cuestionario se pide al estudiante localizar la hipótesis de uno de los experimentos descritos en el texto, y se le pide una interpretación crítica del mismo. Los géneros argumentativos o explicativos científicos, como lo es el artículo de investigación, están formados de secuencias descriptivas, explicativas, argumentativas e, incluso, narrativas, para cumplir la función que dentro del mundo científico cumplen (Stagnaro 2012). A este tipo de textos complejos es a los que se enfrenta al estudiante EMS en estos exámenes y, en general, en su vida académica, por lo que las definiciones textualistas pueden confundir a un estudiante que realmente esté tratando de clasificar el tipo de texto que lee.

Muy cercano a este aspecto, debemos notar que Ciudad entre nubes fue extraído de una revista de divulgación científica comercial muy conocida en nuestro país (Muy interesante), lo cual nos lleva a la reflexión acerca de la pertinencia de este tipo de publicaciones con fines de lucro para usarlos como si fueran textos de estudio, pues, el texto divulgativo en este tipo de revistas no está elaborado para ser estudiado, sino para ser consumido en situaciones menos formales. El carácter comercial de la revista no lo hace ser una fuente ideal para evaluar la lectura académica; dentro de la divulgación científica podrían ser más pertinentes revistas generadas en las instituciones universitarias o asociaciones científicas reconocidas que publican periódicamente este tipo de revistas divulgativas, pues es en estas instituciones en donde los científicos realizan su labor de investigación y los fines de publicación no se relacionan con la venta al público, sino con la difusión del conocimiento.

Además, como ya se mencionó, es necesario que se les enseñen las estrategias adecuadas para resolver tareas como la interpretación y la reflexión, pero particularmente, para que posean una habilidad metacognitiva desarrollada en relación con el registro de la disciplina específica del tema de estudio, porque es situadamente como los lectores toman conciencia de las características particulares de la manera específica en la que la disciplina y tema del texto configuran las opciones sintácticas, semántica y pragmáticas de la lengua, en este caso, un discurso biomédico de especialista, y es cuando pueden comprender mejor (Castro Azuara, et al. 2013b). Para ello, es necesario que los estudiantes realicen el reconocimiento de estas características de registro con el maestro de ciencia, que es el especialista.

Hernándeそ, Rosales y Galicia doi: https://doi.org/10.36799/el.v1i1.11 Volumen 1, Número 1, Año 2016, ISSN: 2448-5942 
En resumen, nos parece que uno de los aspectos de mayor cuidado en este tipo de pruebas es escoger el texto con el cual se evaluará, pues la pertinencia del texto (tema, registro, género, propósito de lectura) para la comunidad lectora que se pretende evaluar, es central para poder obtener resultados significativos. No sólo porque el texto se considere de divulgación puede ser adecuado para utilizarse como instrumento de evaluación a una comunidad escolar heterogénea en muchos sentidos, pues se corre un riesgo real en la construcción de sentido por la falta de vinculación de los lectores con la situación social de lectura (Vaca 2005). De esta forma, como hemos expuesto en otros espacios, una clasificación basada en el género podría mostrar con mayor claridad para ellos cómo con el lenguaje se cumplen las acciones sociales en el contexto académico (Hernández Ramírez 2010b).

\section{ANÁLISIS DE REACTIVOS EN ENLACE 2010}

A continuación presentamos una tabla en la que resumimos los resultados del ejercicio de comprensión lectora del texto expositivo Ciudad entre nubes (Posada-Swafford 2003), aplicada a los estudiantes de Enseñanza de Lenguas de la UAT y de la Ingeniería en Mecatrónica de la UTT y que, como se mencionó anteriormente, pertenece a un ejercicio de prueba disponible en el sitio web oficial de la prueba que simula la prueba que se presentó en el año 2010. En ella registramos el número de pregunta, la localización de la respuesta, el tipo de habilidad que se evalúa y el porcentaje de estudiantes que en nuestro ejercicio acertaron en la respuesta.

Hernández, Rosales y Galicia doi: https://doi.org/10.36799/el.v1i1.11 Volumen 1, Número 1, Año 2016, ISSN: 2448-5942 
Tabla 1. Resultados generales de la prueba ENLACE.

\begin{tabular}{|c|c|c|c|c|c|}
\hline $\begin{array}{l}\text { No. } \\
\text { pregunta }\end{array}$ & Mecanismo estratégico de respuesta & $\begin{array}{l}\text { Respuesta } \\
\text { correcta }\end{array}$ & $\begin{array}{l}\text { Lugar del texto de la } \\
\text { respuesta }\end{array}$ & Nivel de habilidad & $\begin{array}{l}\% \text { Resp. } \\
\text { Correcta }\end{array}$ \\
\hline 94 & Localización información literal & A & Párr. 13 & Extracción & $96 \%$ \\
\hline 95 & \begin{tabular}{|lll} 
Inferencia básica: síntesis y enlace de \\
referentes para deducir el "daño" (conc. \\
Previos)
\end{tabular} & D & Párr. 1 y 4 & Interpretación & $73 \%$ \\
\hline 96 & $\begin{array}{|ll|}\text { Inferencia básica: sintesis y enlace de } \\
\text { referentes para deducir el "daño" (conc. } \\
\text { Previos) }\end{array}$ & D & Párr. 6 y 7 & Interpretación & $38 \%$ \\
\hline 98 & Síntesis, utilizando elem entos de cohesión & D & Párr. 4 y 6 & Interpretación & $68 \%$ \\
\hline 99 & Inferencia compleja. Interpretación & D & $\begin{array}{l}\text { Párr. } 7 / / \text { Hay que leer el } \\
\text { pámafo anterior }(6)\end{array}$ & Interpretación & $20 \%$ \\
\hline 100 & $\begin{array}{|lllll|}\begin{array}{l}\text { Sintesis de las ideas principales } \\
\text { jerarquizarlas }\end{array} & \text { y } \\
\end{array}$ & A & Texto completo & Interpretación & $92 \%$ \\
\hline 101 & Inferencia básica. U bicación en el texto & c & Tabla y glosario & Extracción & $38 \%$ \\
\hline 102 & Inferencia léxica. & B & Tabla & Extracción & $77 \%$ \\
\hline 103 & $\begin{array}{l}\text { Identificación de relación lógica (problema- } \\
\text { solución) }\end{array}$ & c & $\begin{array}{l}\text { Texto com pleto. En la } \\
\text { pregunta misma }\end{array}$ & Interpretación & $27 \%$ \\
\hline 104 & Inferencia por relación sinonímica del léxico & D & Párr. 5 & Extracción & $40 \%$ \\
\hline 105 & Sintesis & c & Párr. 19. Conclusiones & Reflexión & $59 \%$ \\
\hline 106 & Inferencia compleja. Interpretación & c & Todo el texto & Interpretación & $33 \%$ \\
\hline 107 & Localización información literal & c & Párr. 7 & Extracción & $65 \%$ \\
\hline 108 & Localización información literal de datos & D & Tabla & Extracción & $68 \%$ \\
\hline 109 & Relación de datos. Combinación de información & A & Tabla & Interpretación & $72 \%$ \\
\hline 110 & Inferencia compleja. Reflexión & A & Todo el texto & Reflexión & $60 \%$ \\
\hline
\end{tabular}

Fuente: Elaboración propia

Como es posible observar, son seis preguntas cuyos porcentajes en relación a la respuesta correcta, se encuentran por debajo del 60\%, a las que hemos considerado como las preguntas que presentaron una mayor dificultad, a saber: 96, 99, 101, 103, 104 y 106. Por cuestiones de espacio, sólo hablaremos de aquellas con los menores porcentajes.

La pregunta 96 es un ejercicio de complementación en el que el enunciado sintetiza una de las ideas manejadas como verdaderas en el texto. La mayoría de las respuestas se reparten entre el inciso c y el d, como se aprecia en la gráfica 1, en donde esta última respuesta que está en negritas es la correcta, pues es la que complementa adecuadamente las dos partes de la aseveración. Para comprender el motivo de este equívoco, veamos qué se plantea en la pregunta: 
96. La exposición de las madres a altos niveles de contaminantes automotores, especialmente la relativa disminuye el del bebé.

a) Bióxido de carbono, efecto inmune

b) Ozono, tono muscular

c) Benzopireno, daño genético

d) Plomo, desarrollo cognitivo

Pregunta 96 de ENLACE 2010.

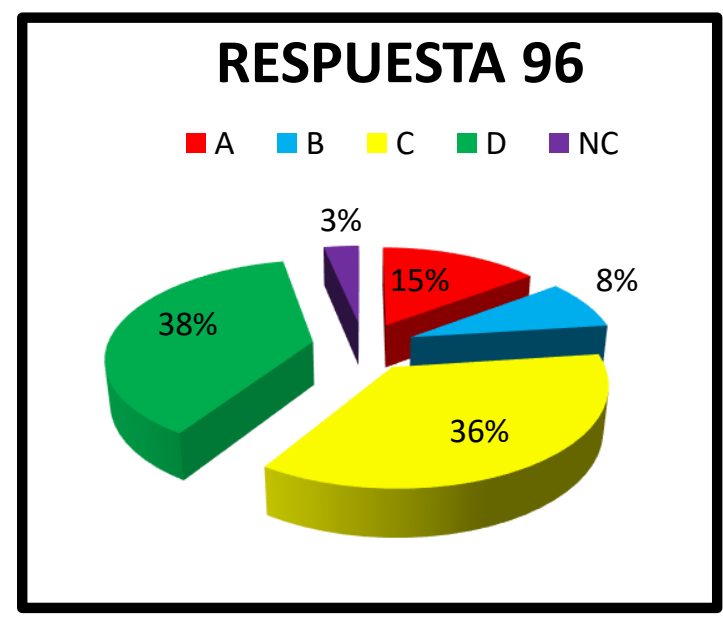

Gráfica 1. Resultados de la pregunta 96

El vocablo Benzopireno es un ítem léxico que es introducido como novedoso para el lector en el texto, por lo que es probable que al reconocer este hecho, el estudiante decida elegirlo como la correcta aunque la segunda parte de la respuesta no se adecue al contexto lingüístico planteado. No realiza, entonces, un proceso de verificación de la coherencia semántica, sino contesta con base en lo que él cree que es lo normal que se pregunte en los exámenes de vocabulario. Es decir, su respuesta se guía en un conocimiento del ritual de este tipo de ejercicios; de esta forma es que evidencia que no se involucra en la construcción de una imagen conceptual del texto.

Por su parte, el reactivo 99 es un ejercicio de síntesis e interpretación, rubro en el que se encuentran los reactivos con menores porcentajes de aciertos, sólo alcanza un 20\% como lo vemos en la siguiente gráfica: 
99. Elija la opción que sintetice la hipótesis de la Dra. Perera sobre las anomalias que la contaminación ambiental provoca en el ADN.

a) La doctora se ha ocupado de medir la exposición de embarazadas a diferentes niveles de la contaminación producida por hidrocarburos aromáticos.

b) Gracias a sus investigaciones, se ha estudiado el ambiente urbano y los cambios casi imperceptibles que ocurren en el organismo humano.

c) Para determinar cómo la contaminación afecta a los fetos, basta con ocupar un monitor de aire que mide las partículas respiradas durante 48 horas.

d) Cuando las partículas son respiradas, llegan a la sangre a través de los pulmones, introduciéndose al cromosoma del feto y produciendo desorden genético.

Pregunta 99 de ENLACE 2010

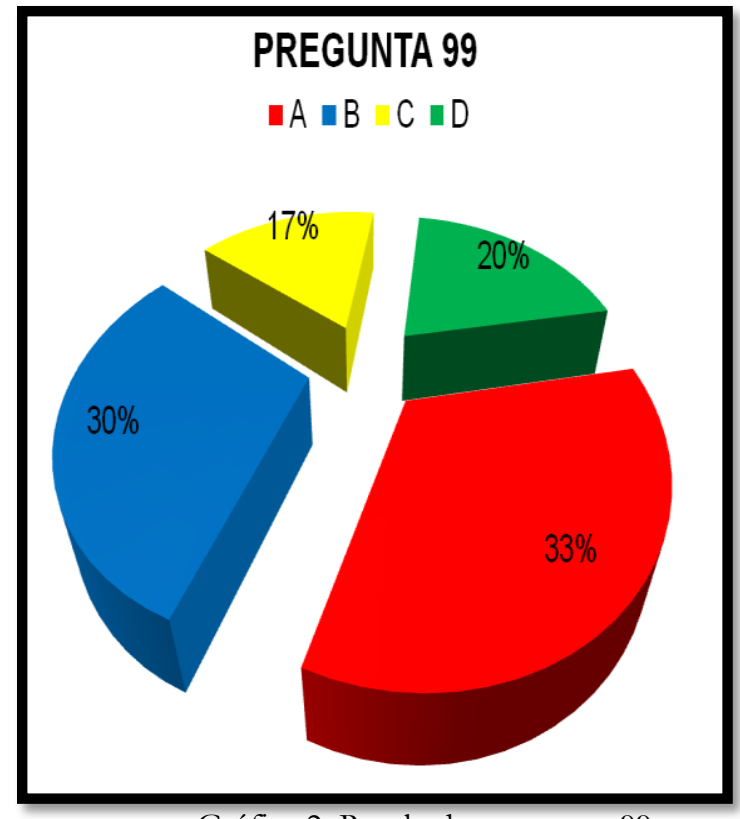

Gráfica 2. Resultados pregunta 99

Esta respuesta requiere que el estudiante sintetice los elementos semánticos que conforman la información dada a los largo de varios párrafos. En las respuestas con mayor incidencia de respuestas se encuentran elementos adjetivos que no corresponden a las acciones descritas en el texto (aromáticos o imperceptibles), sin embargo son escogidas porque son enunciados que describen una idea principal del texto; en cambio, la respuesta correcta parece la descripción de un proceso más que una generalización, sin embargo es la que más se parece a lo expresado en el texto. De esta manera, a partir de la información dada por los estudiantes entrevistados, es posible interpretar que se confunden al no identificar estas sutilezas del texto que tiene que ver con un conocimiento más especializado sobre la materia, por eso, cualquiera de las dos primeras parecen ser mejores respuestas que las demás. A partir de esto, es que podemos corroborar lo dudoso que resulta asumir que habilidad lectora sea independiente de un conocimiento especializado.

La pregunta 103 fue, sin duda, la que representó la de mayor confusión para todos los lectores que contestaron la prueba. Mediante ella, se intenta evaluar la capacidad de los estudiantes de reconocer una relación de tipo semántico o de coherencia (problema-solución) entre las cláusulas señaladas. Los porcentajes de las respuestas nos hacen deducir que la diferencia entre las cuatro opciones no son claras para los estudiantes y, al verificar en el 
diccionario de la RAE, las tres palabras (explicación, ilustración y aclaración) coinciden en tener como propósito disipar la confusión con respecto a un conocimiento. Esto nos dice que no se aclaran en la prueba los conceptos que se piensa como los ideales para las respuestas. Quizá suponen los evaluadores que el reconocimiento de las relaciones de coherencia a través de etiquetas preconcebidas como parte-todo, problema-solución, etc., es transparente para cualquier lector, lo cual nos ilustra de una falla en el diseño del reactivo, no en la habilidad del lector.

\section{3. ¿Qué relación existe entre las siguientes cláusulas? \\ 1. Los niveles de ciertos contaminantes pueden causar una serie de anomalías en el $\mathrm{ADN}$ \\ 2. Las autoridades sanitarias deben determinar si las regulaciones vigentes protegen a los niños en gestación
a) Explicación
b) Ilustración
c) Solución
d) Aclaración

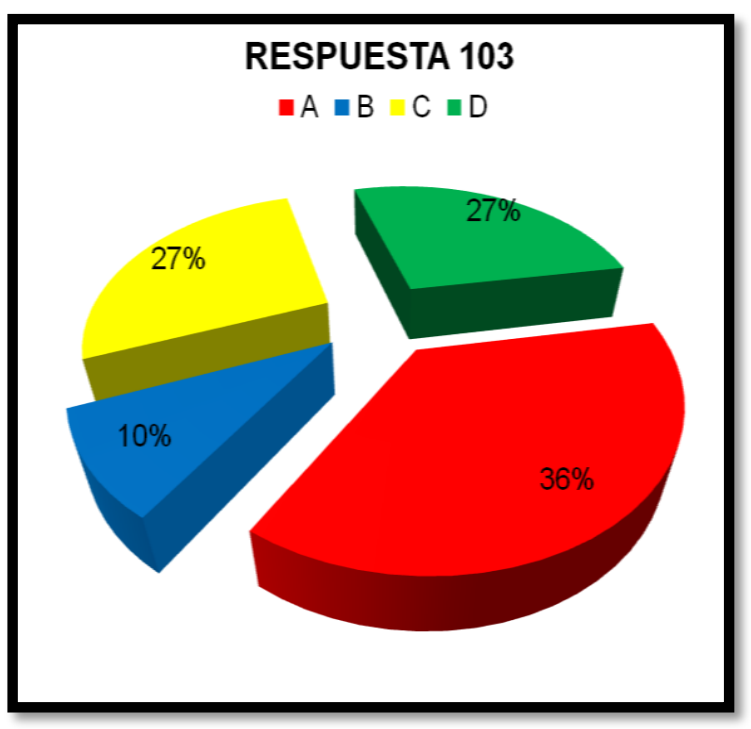

Gráfica 3. Resultados de la pregunta 103

Pregunta 103 de ENLACE 2010

Por último, en la pregunta 106 vemos que los porcentajes de respuestas se concentran en los primero tres incisos; la respuesta correcta es la que tiene mayor relación con el tema del texto, sin embargo, esto último no está aclarado en la pregunta, por lo que los lectores escogen la que a su juicio y a sus conocimientos previos es la mejor respuesta, pues, como podemos apreciar, cada una de las respuestas que se proponen, sin duda alguna, son fenómenos derivados del recalentamiento del aire y de los mares.

De nuevo, aunque podría pensarse que sería innecesaria tal aclaración por obvia, nuestros resultados señalan que sería pertinente incluir la aclaración para poder verificar si esto modifica las respuestas o no, y de ahí podría formularse un juicio más claro para hacer aseveraciones. 
106. El recalentamiento del aire y de los mares por el desequilibrio químico de la atmósfera propicia:

a) Una mayor incidencia en las enfermedades de niños y jóvenes.

b) La amenaza de extinción de especies animales y vegetales.

c) Un incremento en las muertes causadas por intoxicación respiratoria.

d) El aumento de frecuencia de terremotos y maremotos.

Pregunta 106 de ENLACE 2010.

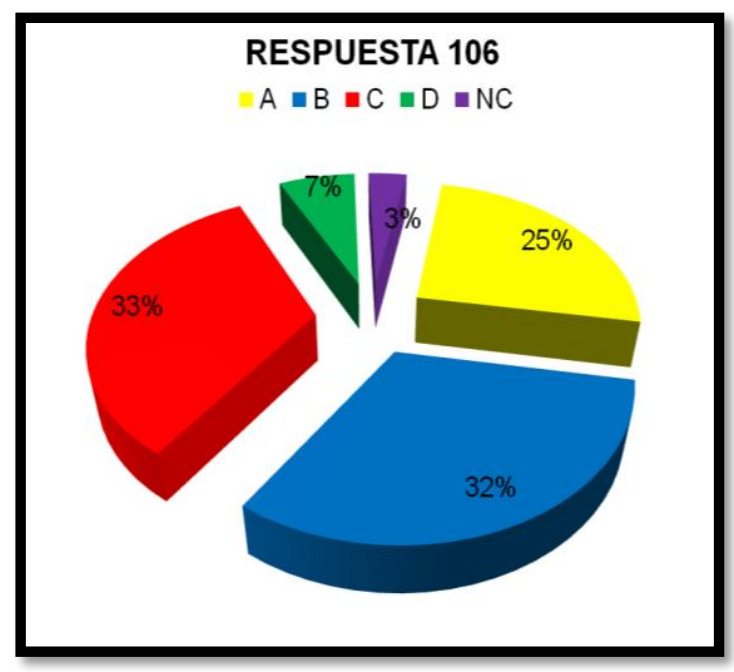

Gráfica 6. Resultados de la pregunta 106.

\section{OBSERVACIONES FINALES}

El punto de vista desde el cual se elabora y evalúa la prueba ENLACE, en donde predomina un concepto cognitivo limitante de la comprensión lectora, pues no integra el componente social dentro de su conceptualización, parece ser un instrumento adecuado para evaluar competencias a nivel de extracción o localización de información, no así para la reflexión y la interpretación, procesos complejos y de un gran componente social e ideológico que, presumiblemente, requiera pruebas cualitativas que sean semejantes a su naturaleza.

Nos interesa hacer énfasis en el carácter especializado y no espontáneo de la comprensión académica especializada; la comprensión como una competencia abstracta no es acorde con los resultados y evidencias de cerca de veinte años de investigación en el campo del lenguaje y la enseñanza de la literacidad.

Aun así, podemos estar de acuerdo en que el carácter diagnóstico de la prueba señala que los estudiantes no están leyendo estratégicamente los textos; sin embargo, esto puede deberse también a diversos factores, no relacionados con las capacidades de los lectores evaluados:

a) Las características del tamaño de la prueba y las circunstancias en las que se aplica.

b) Las condiciones desiguales de literacidad en las que se aplica la prueba. 
c) La organización y secuencialidad de los reactivos en la prueba, pues dificulta el manejo rápido de la información.

d) Las dificultades de los estudiantes para reconocer y manejar estratégicamente el registro científico por parte de los estudiantes.

e) La inexistencia de una sistematización en la enseñanza de estrategias lectoras para la lectura académica especializadas a lo largo del currículo de enseñanza y dentro de las diferentes asignaturas, por lo que el estudiante no las ejerce.

Por todo esto, creemos prematuro y poco confiable ceñirse a los resultados de la prueba para afirmar que los estudiantes poseen o no una comprensión lectora adecuada y manejar a partir de estos parámetros la imagen, ya sea a nivel nacional o internacional, de una comunidad lectora. Es necesario aún discutir por equipos de mayor interdisciplinariedad el alcance, la pertinencia y el potencial de las pruebas para que estas efectivamente sirvan para sistematizar los lineamientos, las prácticas y las políticas públicas relacionadas con la enseñanza de la lengua.

\section{REFERENCIAS BIBLIOGRÁFICAS}

Backhoff Escudero, Eduardo. "Estudio de validez de Enalce: Educación Básica”. Presentación en PPT. (abril, 2014). Consultado el 3 de septiembre de 2014.

<http://www.inee.edu.mx/index.php/organos-colegiadosconferencia-del-sistemanacional-deevaluacion-educativa/1704-documentos-primera-sesion-ordinaria $>$.

Bolívar, Adriana. Análisis interaccional del discurso: del texto a la dinámica social. Análisis del discurso, ¿Por qué y para qué? Venezuela: Los libros de El Nacional y Universidad Central de Venezuela, 2007.

Carlino, Paula. "Alfabetización académica diez años después”. Revista Mexicana de Investigación Educativa. 18. 57. (2013): 355-381.

Castro, María Cristina (coord.); Rosalina Domínguez, Laura Aurora Hernández, Sergio Reyes y Martín Sánchez. Alfabetización académica y comunicación de saberes: la lectura y la escritura en la universidad. México: Universidad Autónoma de Tlaxcala, $2013 a$.

Hernándeæ, Rosales y Galicia doi: https://doi.org/10.36799/el.v1i1.11 Volumen 1, Número 1, Año 2016, ISSN: 2448-5942 
(coord.); Martín Sánchez y Laura Aurora Hernández. Prácticas de escritura académica en la universidad: la producción del ensayo escolar. México: UAT, 2013b.

CENEVAL. Manual Técnico Enlace Media Superior 2008-2010. México: CENEVAL. 2012. Consultado el 11 de julio de 2014. http://www.enlace.sep.gob.mx/content/ms/docs/ 2012/Manual_Tecnico_ENLACE_MS.pdf

- Manual Técnico Enlace Media Superior 2010-2011. México: CENEVAL. 2013. Consultado el 11 de julio de 2014. http://www.enlace.sep.gob.mx/content/ba/docs/ manual_tecnico_enlace10.pdf . Manual para Docentes y Directivos Enlace Media Superior 2014. México: CENEVAL. 11 de julio de 2014. http://www.enlace.sep.gob.mx/content/ms/docs/2014/Manual_Docente_ ENLACEMS_2014.pdf

Díaz, Ángel. "Las pruebas masivas. Análisis de sus diferencias técnicas". Revista Mexicana de Investigación Educativa. 11.29. (2006): 583-615.

Hernández, Laura Aurora. "El Género como concepto organizador del currículum en el Programa 2006 de español en la Escuela Secundaria", Memorias del XI Encuentro Nacional de Estudios en Lenguas, México: Universidad Autónoma de Tlaxcala. 2010a

- "La pedagogía basada en el Género de la lingüística sistémico funcional: Aprender la lengua, aprender con la lengua y aprender sobre la lengua”. Lingüistica Mexicana. V. 1. p. 67-89. 2010b.

Martínez, María Cristina. Lectura y escritura de textos. Perspectivas teóricas y talleres. Cali, Colombia: Cátedra Unesco MECEAL: Lectura, 2002.

Merino, Georgina T. "Leer sin goce". Confabulario, Segunda época. Suplemento cultural de El Universal (México) septiembre, 2013. Consultado el 10 de noviembre de 2014. http://confabulario.eluniversal.com.mx/leer-sin-goce/

Sánchez, Manuel. "Las evaluaciones estandarizadas: sus efectos en tres países latinoamericanos”. Revista Latinoamericana de Estudios Educativos. XLIII. 1. (2013): 97-124.

N.P. Enlace 2013. Información Básica. Subsecretaria de planeación y evaluación de políticas educativas, SEP. Presentación en PDF. (2013). Consultado el 3 de septiembre de 2014. 
http://www.enlace.sep.gob.mx/content/gr/docs/2013/ENLACE_InformacionBasica .pdf

Parodi, Giovanni. Saber leer. México: Instituto Cervantes, Aguilar. 2011.

Posada,-Swafford, A. "Ciudad entre nubes". Muy Interesante 05. Año XXIV, (2003): 34-40. Consultado el 5 de junio de 2013. <http://ENLACE.sep.gob.mx/ba/docs/2010/ ENLACE10_MS.pdf>

Sánchez, Martín. "La exposición y la argumentación en el discurso académico". Prácticas de escritura académica en la universidad: la producción del ensayo escolar. Castro Azuara, María Cristina, (coord.). México: Universidad Autónoma de Tlaxcala (2013): 57-74.

Stagnaro, Daniela. "Negociación del conocimiento en el artículo de investigación en economía: exploración del subsistema de modalización del español de Argentina y lineamientos para una propuesta didáctica". Actas del VIII Congreso de la ALSFAL Lenguaje y Sociedad. Aportes de la Lingüistica sistémico funcional al análisis del discurso verbal y multimodal en la educación y otras disciplinas. Montevideo: Facultad de Ciencias Humanas de la Universidad Católica de Uruguay, 2012. Consultado el 5 de junio de 2014. https://brochagorda. files.wordpress.com/2013/05/viii-congreso-alsfal-libro-de-conferencia.pdf

Vaca, Jorge. Leer. México: Instituto de Investigaciones en Educación, Universidad Veracruzana, 2008.

. "Pisa sin prisa". CPU-e, Revista de Investigación Educativa 1 (julio-diciembre, 2005). Consultado el 5 de octubre de 2014. https://www.uv.mx/cpue/num1/inves/pisa.htm y Benjamín Montiel. "ENLACE 2006: Del constructivismo al regreso de los tests". CPU-e, Revista de Investigación Educativa, 6 (2008): 1-48. MLA International Bibliography. Redalyc. http://www.redalyc.org/pdf/2831/283121716003.pdf

Vidal, Rafael. ¿ENLACE, Exani, Excale o Pisa? México: CENEVAL, 2009.

Zanotto, Mercedes y Gabriela González. "Evaluación del lenguaje en el ámbito escolar”. Lenguaje y Educación. Temas de investigación educativa en México. Carrasco Altamirano, Alma y López-Bonilla, Guadalupe (coord.). México: Fundación SM, Consejo Puebla de Lectura y Ceidea, (2013): 199-238. http://www.inaoep.mx/ cplorg/pdfs/lye.pdf

Wenger, Etienne. Comunidades de práctica. Barcelona: Paidós, 2011. 